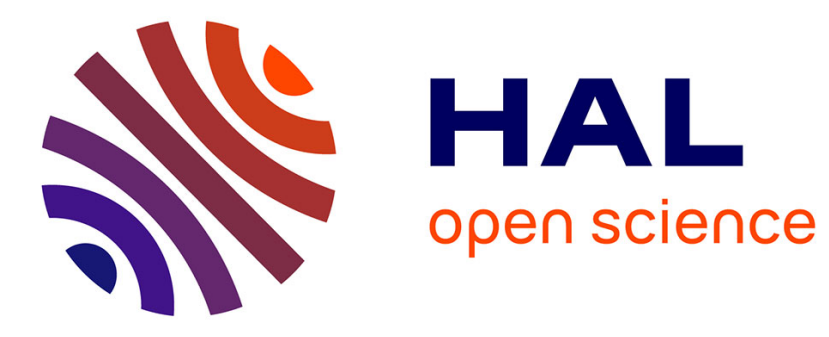

\title{
Delay Estimation for DVB-T Signals in Adverse Multipath Scenarios
}

Liang Chen, Elena Simona Lohan, Olivier Julien, Paul Thevenon, Christophe Macabiau, Laura Ruotsalainen, Heidi Kuusniemi

\section{To cite this version:}

Liang Chen, Elena Simona Lohan, Olivier Julien, Paul Thevenon, Christophe Macabiau, et al.. Delay Estimation for DVB-T Signals in Adverse Multipath Scenarios. ICL-GNSS 2014 International Conference on Localization and GNSS, Jun 2014, Helsinki, Finland. pp.1-6, 10.1109/ICLGNSS.2014.6934167 . hal-01158737

\section{HAL Id: hal-01158737 \\ https://hal-enac.archives-ouvertes.fr/hal-01158737}

Submitted on 1 Jun 2015

HAL is a multi-disciplinary open access archive for the deposit and dissemination of scientific research documents, whether they are published or not. The documents may come from teaching and research institutions in France or abroad, or from public or private research centers.
L'archive ouverte pluridisciplinaire HAL, est destinée au dépôt et à la diffusion de documents scientifiques de niveau recherche, publiés ou non, émanant des établissements d'enseignement et de recherche français ou étrangers, des laboratoires publics ou privés. 


\section{Delay Estimation for DVB-T Signals in Adverse Multipath Scenarios}

\author{
Liang Chen \\ Department of Navigation and Positioning \\ Finnish Geodetic Institute \\ Masala, 02431, Finland \\ Email:liang.chen@fgi.fi
}

\author{
Elena Simona Lohan \\ Department of Electronics and Communication Engineering \\ Tampere University of Technology \\ Tampere, 33720, Finland \\ Email: elena-simona.lohan@tut.fi
}

Laura Ruotsalainen, Heidi Kuusniemi

Department of Navigation and Positioning

Finnish Geodetic Institute

Masala, 02431, Finland

Email: \{laura.ruotsalainen,heidi.kuusniemi\}@fgi.fi

\begin{abstract}
Timing approaches, such as time of arrival (TOA) and time difference of arrival (TDOA), employed with DVB-T are sensitive to the presence of multipath errors. This paper studies the delay estimation in adverse multipath scenarios using the DVB-T signals. A datadirected delay tracking mechanism is presented for signal tracking to estimate the time delay. Three different delay locked loops (DLLs) are considered to mitigate the multipath errors, i.e. the early minus late (EML), the narrow EML (nEML) and the High Resolution Correlator (HRC). Simulation results are compared for tracking the DVB-T signals in the suggested Rice and Rayleigh channels. Simulation results show that the correlation outputs of the DVB-T signals are relatively smooth, which cause the DLLs difficulty to detect the closely spaced multipaths. For tracking the first peak of the correlation, HRC and nEML are better than EML.
\end{abstract}

\section{INTRODUCTION}

Global Navigation Satellite Systems (GNSS) have been put into use in mass-market applications in recent years. However, as it is well known, the signal availability and the positioning accuracy dramatically deteriorate when these systems are operated in challenging environments, such as indoors and urban canyons [1], [2], [3]. Recently, digital broadcasting systems, such as Digital Video Broadcasting (DVB), Digital Audio Broadcasting (DAB), and the ATSC, have been widely used as an alternative information transmission technique [4]. It has been recognized that novel wireless location methods can be designed by utilizing the digital broadcasting signals [5]. Compared with the GNSS, wireless localization based on the digital broadcasting has a range of potential advantages: the signal transmission power is stronger and the frequency band is within $300-900 \mathrm{MHz}$, which contribute to better diffraction performance than the currently used GPS L1 or Galileo E1 of $1.5 \mathrm{GHz}$. Therefore, a better receiving quality indoors is expected [6]. The nominal signal bandwidth of the terrestrial digital TV broadcasting is designed between $6-8 \mathrm{MHz}$, which is much larger than the chipping rate of GPS L1 or Galileo E1 signals. This will improve the precision of timing. The digital TV (DTV) signals are transmitted continuously and the location of the DTV transmitters are fixed. In contrast to the GNSS, the range between the DTV transmitters and the receivers changes very slowly. Therefore, the DTV signal does not experience significantly the Doppler effects and the impairment caused by the delay of ionosphere propagation, which will lead to easier signal acquisition and the possibility of integration over a longer period of time [5]. As a signal of opportunity (SoO) for positioning, the DTV facilities have already been in use and no more infrastructure investment is required, except the position devices.

Research interest in positioning using DTV systems has grown rapidly. Basically, these methods can be divided into two categories: methods for single-carrier ATSC system [5], [7], [8] and methods for multicarrier Orthogonal Frequency Division Multiplex (OFDM) system [9], [10], [11], [12], [13], [14], [15], [16], [17], [18], [19]. The ATSC, as a single-carrier modulation system, is vulnerable to multipath fading, while digital broadcasting based on OFDM systems have been tested to mitigate the effect of multipath fading more effectively [20]. As the standards based on OFDM modulation, e.g. DVB-T/H, T-DMB, etc, have been widely adopted in most countries, we may predict that the wireless position systems based on the multi-carrier OFDM have massive potential usage in the future.

For the purpose of high accuracy positioning and navigation with the OFDM signals, there are several methods to determine the time of arrival (TOA) proposed in the literature. One of them is based on a sliding correlator for the coarse timing acquisition by using the property of OFDM cyclic prefix [21]. Another method is to use Delay-locked loop (DLL) or early-minus-late (EML) loop for a finer timing 
tracking [22]. Scatter pilots have been used as the local template in the DLL [9], [10], [11]. In authors' previous work [15], full OFDM symbols demodulated from the receiver were further used to produce the copies of the transmitted OFDM signals and applied into the DLL.

However, in dense urban scenarios and indoors, it is common that signals are propagated in multipath environment. Multipath imposes positive biases on the delay of the line of sight (LOS) path and is therefore the main source for range-based positioning techniques, such as TOA or time difference of arrival (TDOA). The classical EML fails to cope with the multipath propagation, especially in closely spaced path scenarios [1]. In GNSS, several enhanced EMLbased techniques have been introduced [2], [3], [23].

In this work, we extend our work in [15] and investigate delay estimation for DVB-T signals in multipath scenarios. Two enhanced delay tracking loops are considered, i.e., the narrow correlator EML (nEML) and the HRC. In GNSS, the nEML improves the noise sensitivity and multipath robustness, while HRC [24] has good noise and multipath resistance, especially for those with several correlation peaks. The performances of these DLLs are compared in the channels for fixed users and for portable mobile devices suggested by ETSI [20].

The rest of the paper is organized as follows: Section II introduces in brief the ETSI DVB-T system. Section III presents the signal model, including OFDM signals plus channel effect. Section IV presents the datadirected time delay estimation methods for multipath mitigation. Simulation results are shown in Section V. Finally, some conclusions are drawn in Section VI.

\section{OFDM SIGNALS IN ETSI DVB-T SYSTEM}

The DVB-T is an European standard for digital TV broadcasting to fixed and portable receivers [20]. OFDM modulation is used in DVB-T. In principle, OFDM is a method of encoding digital data on multiple (sub)carrier frequencies. The width of these subcarriers is chosen narrow enough so that the channel frequency response can be considered as flat over the subcarrier bandwidth. Meanwhile, these subcarriers are kept in orthogonality and overlapped without interfering with each other, which achieves an excellent spectral efficiency and no Inter-Carrier Interference (ICI) when the receiver is synchronized. In addition, to avoid InterSymbol Interference (ISI), a guard interval is inserted before the useful OFDM symbols. In DVB-T, this guard interval is a replica of the last several tens of samples of the OFDM symbol, which is also referred to as Cyclic Prefix (CP).

In DVB-T, signals are transmitted in stream with a fixed rate, which is suitable for continuous tracking. The transmitted signal is organized in frames. One
OFDM frame consists of 68 OFDM symbols. In each OFDM symbol, there are different types of subcarriers, i.e., null subcarriers, which have zero values and are situated on the edges of the signal spectrum and serve as guard bands to avoid out-of-band emissions of the OFDM signal, data subcarriers, which are the payload information to be transmitted, Transmission Parameter Signaling (TPS) subcarriers, which carry information about the transmission parameters and pilot subcarriers, which are given by a known PseudoRandom Binary Sequence (PRBS) and used mainly for synchronization, channel estimation and equalization.

The parameter settings of these subcarriers are based on different modes suggested by ETSI [20]. It is also noted that, in DVB-T, different emitters are required to be coordinated to the GPS time and to transmit the DTV signals at the same time in a single frequency network [20]. This network synchronization makes the pseudorange measurements available in practice for mobile tracking.

\section{THE RECEIVED BASEBAND SIGNAL WITH CHANNEL IMPAIRMENT}

Here, we consider the ideal transmitted baseband OFDM signal, which can be expressed as

$$
\begin{gathered}
s(k)=\frac{1}{\sqrt{N_{\mathrm{FFT}}}} \sum_{n=0}^{N_{u}-1} S(n) \exp \left(j \frac{2 \pi k n}{N_{\mathrm{FFT}}}\right), \\
k=0,1, N_{\mathrm{FFT}}+N_{\mathrm{GI}}-1
\end{gathered}
$$

where $\{S(n)\}$ are the modulated data symbols for the $N_{u}$ used DVB-T subcarriers, $N_{\mathrm{FFT}}$ is the FFT length and $N_{\mathrm{GI}}$ is the number of samples for the guard interval.

As a typical wireless transmission system, DVB-T signals usually travel along multiple paths, arising from reflection, scattering and diffraction, which are due to numerous obstacles in the propagation environment. Suppose that the signal $s(k)$ is transmitted over a frequency selective fading channel of length $L$ with path gains $h(l)$, where $0 \leq l \leq L-1$, the signal becomes

$$
x(k)=\sum_{l=0}^{L-1} h(l) s(k-l)+n(k), k=0,1, \cdots,
$$

where $n(k)$ is the additive white Gaussian noise (AWGN) with zero mean and variance $\sigma_{n}^{2}$. At the receiver, the received signals experience symbol-timing offset (STO), carrier-frequency offset (CFO), and sampling clock offset (SCO) [25]. When taking these into account, the received samples can be written as

$$
r(k)=\exp \left(j \frac{2 \pi k \epsilon}{N_{\mathrm{FFT}}}\right) x(k-\theta)
$$

where $\theta$ is the STO in terms of OFDM samples, $\epsilon$ assumed CFO normalized by the subcarrier spacing. The timing point of the start of the FFT window is determined by the timing synchronization to be at the sample $r(\theta)$. 


\section{DATA DIRECTED DELAY ESTIMATION FOR MULTIPATH MITIGATION}

The data directed delay estimation is divided into two parts: the communication part, which is to demodulate the transmitted OFDM symbols, and the navigation part, which is to track the signals to obtain the TOA. To mitigate the multipath errors, different delay tracking methods are adapted in the navigation part.

\section{A. Data demodulation}

The data demodulation can be further divided into coarse symbol synchronization, channel estimation and equalization.

1) Coarse symbol synchronization: In DVB-T receivers, the correlation method based on guard interval is employed to acquire the coarse symbol synchronization. The STO $\theta$ and CFO $\epsilon$ can be jointly estimated by the maximum likelihood (ML) algorithm derived in [21]. The estimated $\hat{\theta}_{\mathrm{ML}}$ is used as the FFT window controller initial value for further data demodulation processing. However, this coarse symbol timing estimation is biased and has a large standard deviation [26].

2) Channel estimation and equalization: In order to achieve fine synchronization, accurate estimation of the channel impulse response (CIR) is required. The least-square based algorithm [27] adapted in [28] is a straightforward method for channel estimation. Furthermore, the residual symbol timing error of the first strong path $\hat{e}_{\theta}$ can be estimated according to the channel power delay profile $\hat{P}_{k}$, which can be used to adjust the start position of the FFT windows, when the fine symbol timing estimation is started [22].

To demodulate the data message, the equalization is utilized to compensate the residual timing and frequency error. Thus, in terms of reliable data demodulation, by applying the above synchronization and equalization steps, the residual timing and frequency errors are small enough for the data demodulation.

\section{B. Data directed delay estimation}

For reliable communications, the OFDM systems have stringent requirements on the timing and frequency synchronization. However, in order to achieve high accuracy positioning, it is necessary to achieve an even finer synchronization, which results in a finer time delay estimation of the received signals

Owing to the employment of powerful error-control coding, it is reasonable to assume that data demodulation is error free. Therefore, the demodulated data can be used to produce the copies of the transmitted OFDM signals The regenerated copies of transmitted OFDM signals are then applied to the fine estimation of the delay. The whole scheme is shown in Figure 1 [15].

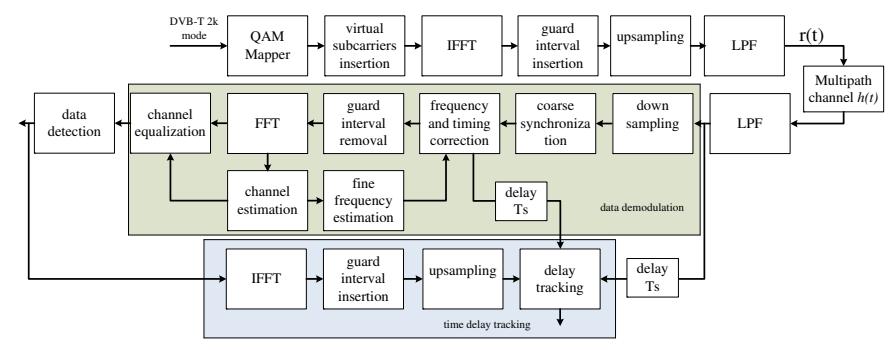

Fig. 1. Delay tracking of OFDM signal

\section{Delay estimation methods in adverse multipath scenarios}

The multipath delay estimation block can be implemented via a feedback loop. The most common feedback structures for the delay estimation are the socalled DLLs. In this work, the following DLLs are investigated in the presence of multipaths.

1) EML: Classical DLL, also called EML correlator consists of 3 correlators (in-prompt, early and late) and adjust the error according to the error between early and late correlators. The early-late spacing has been traditionally 1 chip for GNSS signals. In DVB-T, the time of 1 chip is equal to the inverse of the whole bandwidth that one channel of DVB-T signal occupies. In [15], a basic DLL is applied in a single path channel to show the feasibility of the method.

2) $n E M L$ : It has been observed in GNSS that narrower spacing (narrow correlator) improves the noise sensitivity and multipath robustness. The choice of correlator spacing usually depends on the receiver's available front-end bandwidth and the associated sampling frequency. In this work, a nEML-based DVB-T signal tracking method will be evaluated.

3) HRC: The HRC loops introduce two extra correlator (one very early, another one very late) in order to better check that the prompt reference signal is aligned with the main peak of the correlation function. In HRC, the discriminator output is formed via:

$$
R_{E}^{2}-R_{L}^{2}-a *\left(R_{V E}^{2}-R_{V L}^{2}\right)
$$

where $a$ is traditionally set as 0.5 . The HRC is proposed to cope with the side-lobes of the autocorrelation (ACF) function for Galileo and modernized GPS signals. As shown in Section V, the ACF of the DVB-T signals also has many side-lobes. We will compare the HRC with the other two DLLs in this work.

\section{Simulation RESUlts}

In this section, simulation results are presented. We use $8 \mathrm{k}$ mode of the DVB-T signals, which is the most common mode put into operation in the European countries. Table 1 gives the parameters used in this work. Based on Table 1, other parameters are well defined according to the ETSI DVB-T standard [20]. 
TABLE I

\begin{tabular}{lr}
\multicolumn{2}{c}{ PARAMETERS FOR SIMULATIONS } \\
\hline parameters & value \\
\hline FFT size (mode) & $8 \mathrm{k}(8192)$ \\
chip rate & $64 / 7 \approx 9.145 \mathrm{MHz}$ \\
CP ratio & $1 / 8$ \\
number of information carriers & 6817 \\
modulation of payload information & $64 \mathrm{QAM}$ \\
\hline
\end{tabular}

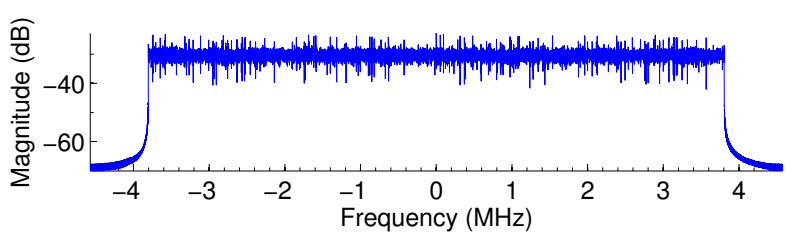

Fig. 2. Spectrum of DVB-T $8 \mathrm{k}$ mode $8 \mathrm{M}$ bandwidth

The spectrum of an ideal DVB-T signal with $8 \mathrm{k}$ mode $8 \mathrm{MHz}$ (effective) bandwidth is shown in Figure 2, where the null margins to avoid the out-ofband emissions and pilot subcarriers that have more power than the payload data are clearly presented. The corresponding ideal ACF is shown in Figure 3. As a comparison, the ACF of GPS C/A code and the Galileo E1 code are also presented. To observe the influence of multipath on delay estimation, the multipath error envelop (MEE) of DVB-T and GPS C/A signals are shown in Figure 4. In Figure 4, the maximum negative and positive errors on delay estimation is computed by setting the delay of one multipath of amplitude equal to half of the amplitude of the LOS signal.

From Figure 3, due to the wider bandwidth the DVB$\mathrm{T}$ signals occupy, the mainlobe in the ACF of DVB$\mathrm{T}$ signal is much narrower (about $\frac{1}{8}$ ) than for GPS L1 C/A and GAL E1, which suggests the increased possibility of detecting two multipaths when their arrival time is larger than one chip of the DVB-T signal (about $0.11 \mu \mathrm{s}$ ). However, by observing the peak of three ACFs, it is also obvious that the peak of the DVB-T signal is smoother than the other two. Therefore, it will be more difficult for DVB-T signals to differentiate two closely spaced multipaths, if they are separated by less than $0.11 \mu \mathrm{s}$. Such inference is in accordance with the results from Figure 4, where the MEE of DVB-T is much smaller than that of the GPS C/A code when multipath is larger than 1 DVB-T chip, while the MEE of DVB-T is slightly larger when multipath is within the distance of 1 chip of the DVB-T signal. It should be noted that the previous statement is true when considering an infinite bandwidth for GNSS receivers. However, although GNSS signals are emitted on a large bandwidth, typically $20 \mathrm{MHz}$ for GPS L1 $\mathrm{C} / \mathrm{A}$, in reality, the front-ends of the GNSS receiver have a limited RF bandwidth, as low as $2 \mathrm{MHz}$ for low-cost receivers, which will smooth the peak of the ACF. On the other hand, this is not true for DVB$\mathrm{T}$ receivers, because DVB-T signals have a strictly limited bandwidth.

To evaluate the performance of three DLLs in the mul-

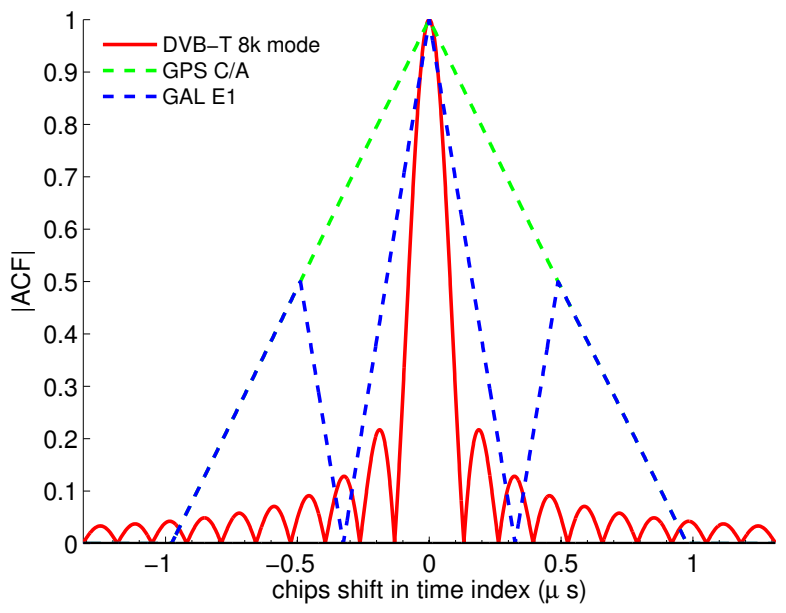

Fig. 3. Ideal ACF of DVB-T, GPS C/A and GALILEO E1

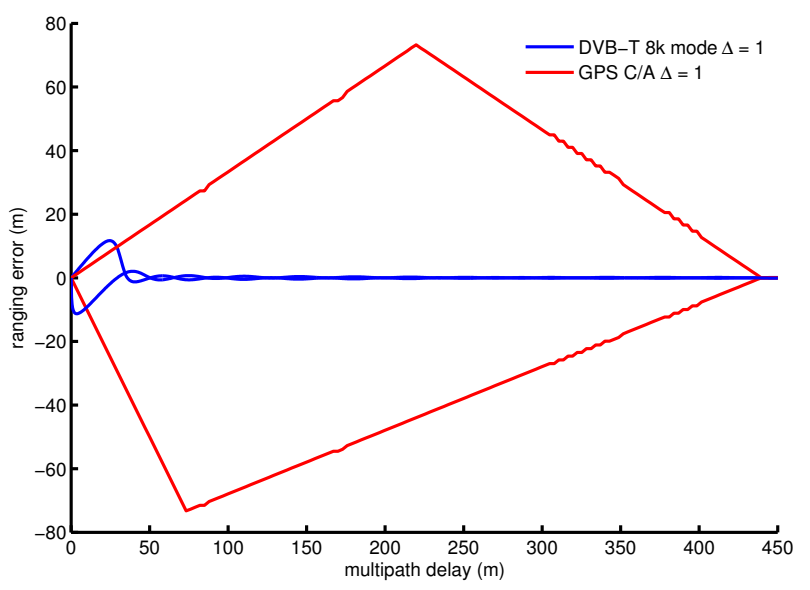

Fig. 4. Multipath error envelope of EML for DVB-T and GPS C/A

tipath scenarios, we use two channel models suggested by the DVB-T standard [20], i.e. a Rice channel model (F1), which is derived from the receivers with fixed position and a Rayleigh channel model (P1), the statistics of which is obtained from the receivers moving with slow speed. They both have 20 multipaths, while the Rice model has an extra LOS component. Figure 5 shows the spectrum of the DVB-T signals experienced in different channel models. The signal noise ratio (SNR) is 10dB. Figure 6 and Figure 8 show channel taps and the correlation output of such DVB-T signals. The corresponding tracking results of the three DLLs are represented in Figure 7 and Figure 9.

From Figure 6 in F1 model, the LOS component has much larger power than the rest of the multipaths (20 times larger suggested by the ETSI standard). But, due to the effect of the multipaths, the first correlation peak has slightly biased and situated on -0.04 chip, which is equivalent to -1.31 meter. Figure 8 shows the P1 model and the signals' correlation output. Without the strong LOS path, the first correlation peak is caused by the first arrived several multipaths, which are closely spaced. The peaks are all smooth and the value of the first peak is on the 0.675 chip, equivalent to $22.15 \mathrm{~m}$. 


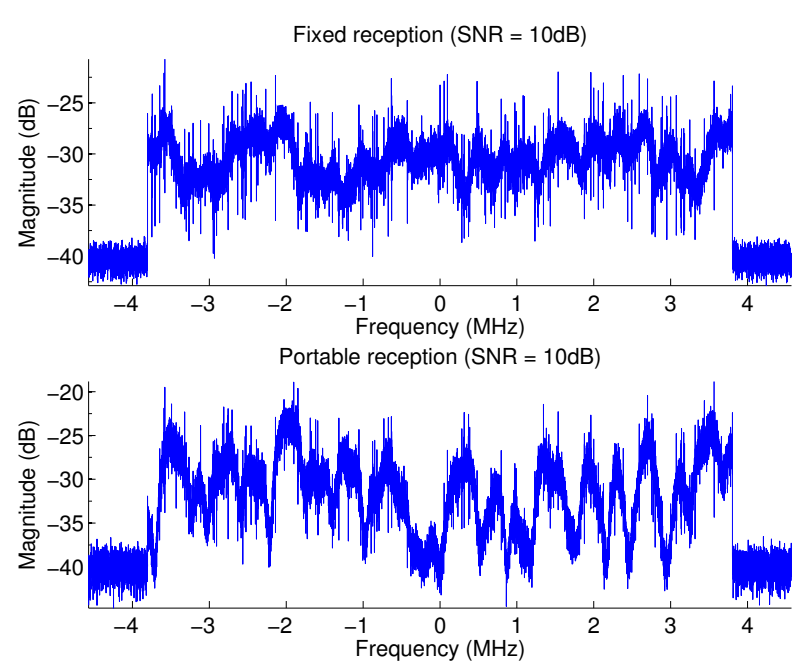

Fig. 5. Spectrum of a 8k mode DVB-T signal in different scenarios

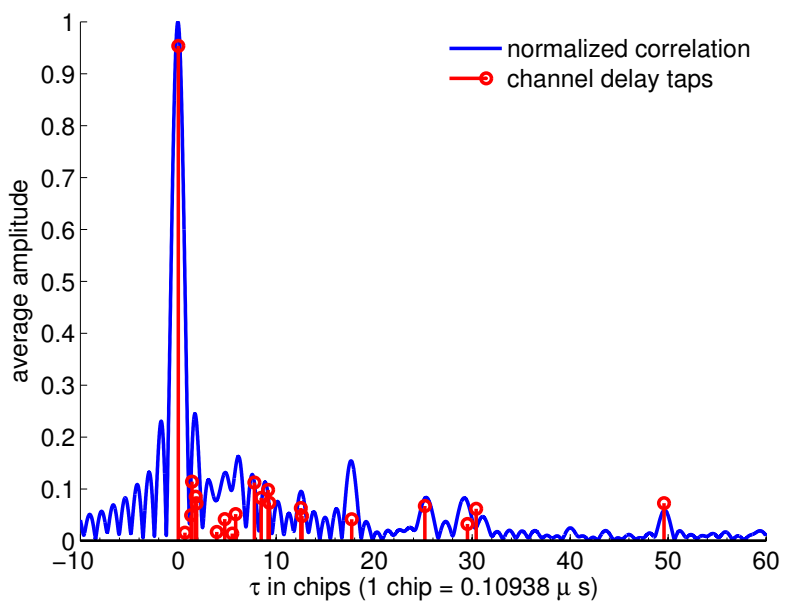

Fig. 6. Channel model of F1 and the correlation output $(\mathrm{SNR}=$ $10 \mathrm{~dB})$

From the tracking results in Figure 7 and Figure 9, the biased peaks of the correlation output cause the tracking errors of all the three DLLs converged to bias errors. However, in term of the performance of tracking the first peak, it is clear the HRC achieves the best, while the nEML has slightly better than EML. Figure 10 gives the statistical results of the tracking errors of the three DLLs. The errors are calculated by using the converged tracking value minus the TOA of the first arrived signal path and the results are averaged in 10 tests in each SNR scenario. In Figure 10, when the SNR is above the requirement for the nearly errorfree demodulation, which is about $-5 \mathrm{~dB}$, the tracking accuracy did not improve with the SNR.

From the results in Figure 7 to 10 , it is clear that, the correlation output of the DVB-T signals are relatively smooth, which causes the closely spaced multipaths hard to be differentiated. The three DLLs are all affected by the peak of the correlation output. In terms of the performance of tracking the first peak, by comparison, the HRC achieves the best, while the nEML has slightly better than EML.

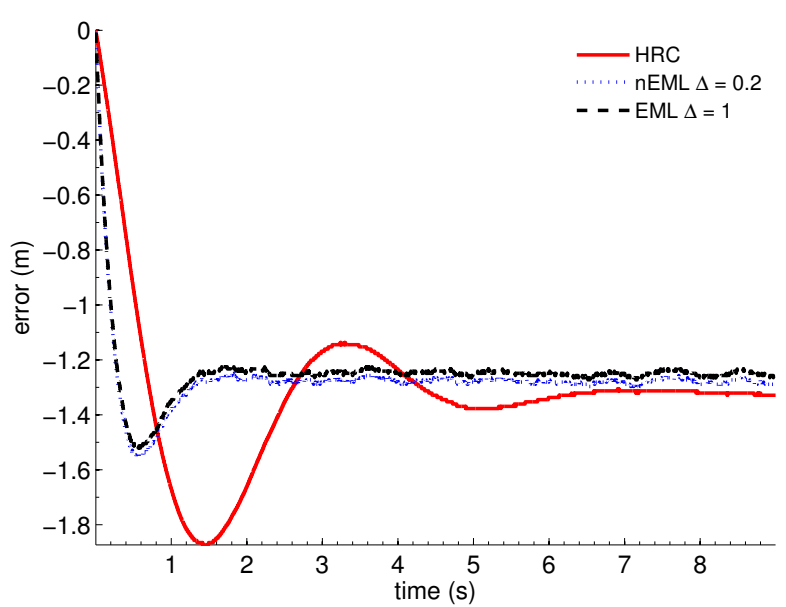

Fig. 7. Tracking results in DVB-T F1 model

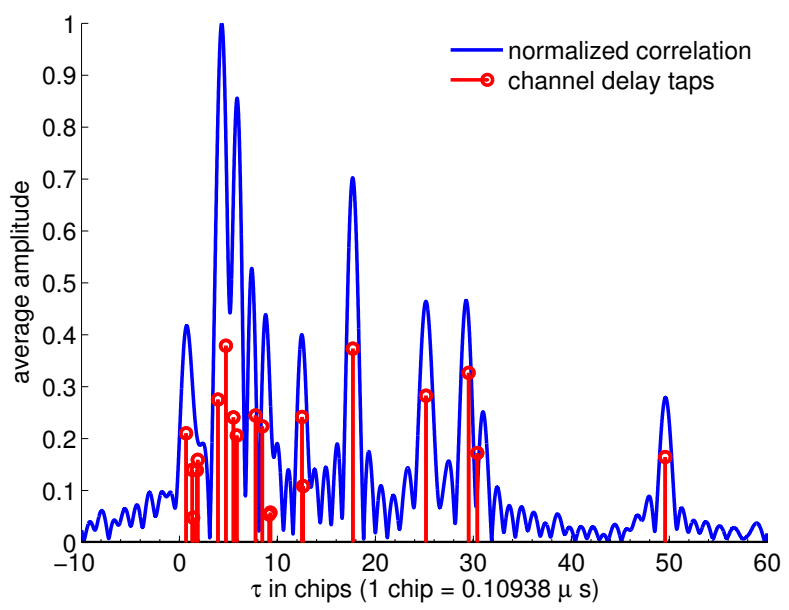

Fig. 8. Channel model of P1 and the correlation output $(\mathrm{SNR}=$ $10 \mathrm{~dB})$

\section{CONCLUSIONS}

This paper studies the delay estimation in adverse multipath scenarios using the DVB-T signals. A datadirected delay tracking mechanism is presented for signal tracking to estimate the time delay. The ACF and MEE suggests much less ranging errors when multipath delays are more than one chip of the DVB-T time duration by comparison with GNSS signals. Three different feedback tracking methods, i.e. EML, nEML and HRC are considered to mitigate the multipath errors. Simulations in the suggested F1 and P1 models showed that, the correlation outputs of the DVB-T signals are relatively smooth, which cause all three DLLs difficult to detect the closely spaced multipaths. For tracking the first peak of the correlation, HRC has showed the best results in multipaths compared with the other tested structures.

\section{ACKNOWLEDGMENT}

This work is partly supported by WIPINGDTV project (No.254232) and CALM project (No. 250266) funded by Academy of Finland. 


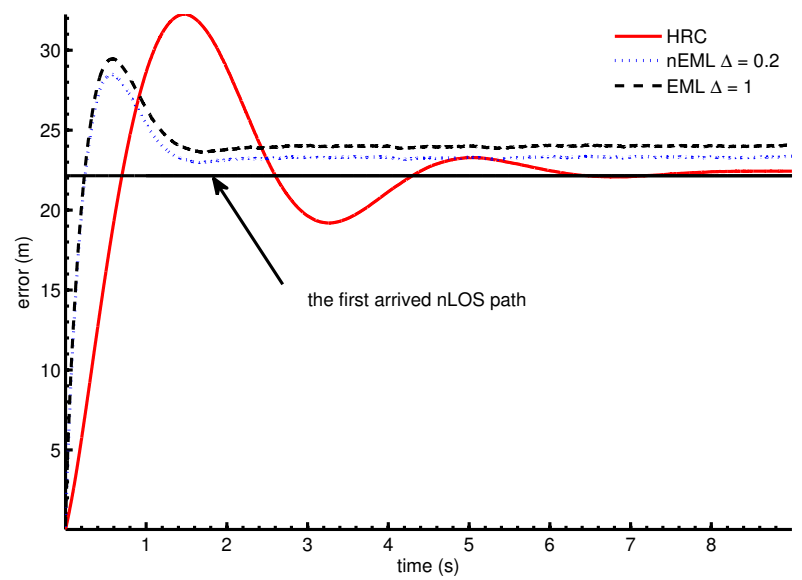

Fig. 9. Tracking results in DVB-T P1 model $(\mathrm{SNR}=10 \mathrm{~dB})$

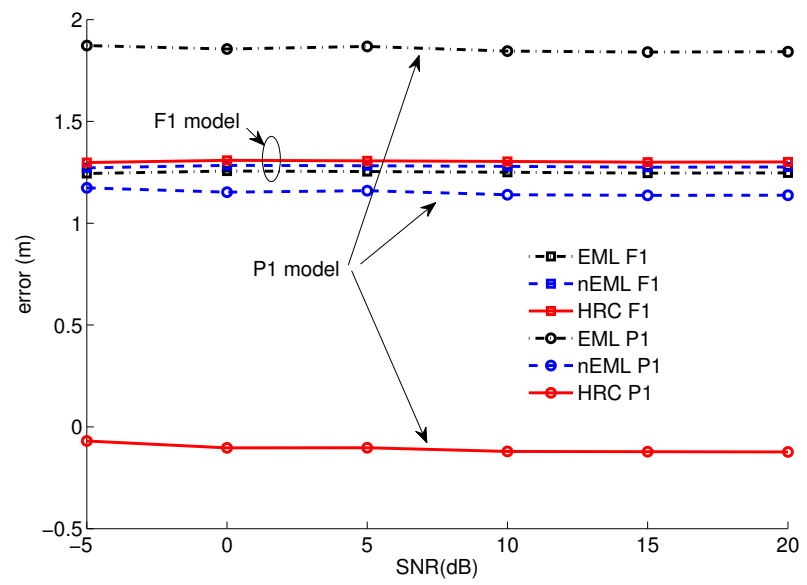

Fig. 10. Tracking errors in DVB-T F1 and P1 models

\section{REFERENCES}

[1] E. D. Kaplan, Ed., Understanding GPS: Principles and Applications. Norwood: Artech House, 1996

[2] E. S. Lohan, A. Lakhzouri, and M. Renfors, "Feedforward delay estimators in adverse multipath propagation for Galileo and modernized GPS signals," EURASIP Journal on Advances in Signal Processing, vol. 2006, no. 1, pp. 1-19, 2006.

[3] H. Hurskainen, E. S. Lohan, X. Hu, J. Raasakka, and J. Nurmi, "Multiple gate delay tracking structures for GNSS signals and their evaluation with Simulink, SystemC, and VHDL," International Journal of Navigation and Observation, vol. 2008, pp. 1-17, 2008.

[4] Y. Wu, S. Hirakawa, U. H. Reimers, and J. Whitaker, "Overview of digital television development worldwide," Proceedings of the IEEE, vol. 94, no. 1, pp. 8-21, Jan 2006.

[5] M. Rabinowitz and J. Spilker, J.J., "A new positioning system using television synchronization signals," IEEE Transactions on Broadcasting, vol. 51, no. 1, pp. 51 - 61, march 2005.

[6] J. T. Ong, H. Yan, S. V. Rao, and G. Shanmugam, "Indoor DTV reception: measurement techniques," IEEE Transactions on Broadcasting, vol. 50, pp. 192-199, 2004.

[7] X. Wang, Y. Wu, and B. Caron, "Transmitter identification using embedded pseudo random sequences," IEEE Transactions on Broadcasting, vol. 50, no. 3, pp. 244-252, September 2004.

[8] X. Wang, Y. Wu, and J. Chouinard, "A new position location system using DTV transmitter identification watermark signals," EURASIP Journal on Applied Signal Processing, vol. 2006, pp. 1-11, 2006.

[9] P. Thevenon, O. Julien, C. Macabiau, D. Serant, L. Ries, S. Corazza, and M. L. Boucheret, "Positioning principles with a mobile TV system using DVB-SH signals and a single frequency network," in Proc. Digital Signal Processing, (DSP09), Santorini, Greece, 5-7 July 2009.
[10] D. Serant, P. Thevenon, M. L. Boucheret, O. Julien, C. Macabiau, S. Corazza, M. Dervin, and L. Ries, "Development and validation of an OFDM/DVB-T sensor for positioning," in Proc. IEEE/ION Position Location and Navigation Symposium (PLANS10), IndianWells, USA, 4-6 May 2010, pp. 998-1001.

[11] D. Serant, O. Julien, C. Macabiau, L. Ries, P. Thevenon, M. Dervin, and M. L. Boucheret, "Positioning using OFDMbased digital TV: new algorithms and tests with real signals," in Proc. 24th International Technical Meeting of the Satellite Division of The Institute of Navigation, Portland OR, USA, 19-23 September 2011, pp. 3451-3460.

[12] D. Serant, O. Julien, L. Ries, P. Thevenon, and M. Dervin, "The digital tv case: Positioning using signals-of-opportunity based on ofdm modulation," Inside GNSS, pp. 54-62, 2011.

[13] L. Dai, Z. Wang, C. Pan, and S. Chen, "Wireless positioning using TDS-OFDM signals in single-frequency networks," IEEE Transactions on Broadcasting, vol. 58, no. 2, pp. 236-246, May 2012.

[14] J. Yang, X. Wang, M. Rahman, S. I. Park, H.-M. Kim, and $\mathrm{Y}$. Wu, "A new positioning system using DVB-T2 transmitter signature waveforms in single frequency networks," IEEE Transactions on Broadcasting, vol. 58, no. 3, pp. 347-359, 2012.

[15] L. Chen, L.-L. Yang, and R. Chen, "Time delay tracking for positioning in DTV networks," in Proc. Ubiquitous Positioning, Indoor Navigation, and Location Based Service (UPINLBS), 2012, Oct. 2012, pp. 1-4.

[16] J. Yan and L. Wu, "A passive location system for single frequency networks using digital terrestrial TV signals," European Transactions on Telecommunications, vol. 22, no. 8, pp. 487499, 2011.

[17] J. Huang and L. Lo Presti, "Dvb-t positioning with a one shot receiver," in 2013 International Conference on Localization and GNSS (ICL-GNSS), June 2013, pp. 1-5.

[18] J. Huang, L. Lo Presti, and R. Garello, "Digital video broadcast-terrestrial (DVB-T) single frequency networks positioning in dynamic scenarios," Sensors, vol. 13, pp. $10191-$ $10218,2013$.

[19] L. Chen, R. Piché, H. Kuusniemi, and R. Chen, "Adaptive mobile tracking in unknown non-line-of-sight conditions with application to digital TV networks," EURASIP Journal on Advances in Signal Processing, vol. 2014, no. 1, pp. 1-22, 2014. [Online]. Available: http://asp.eurasipjournals.com/content/2014/1/22

[20] ETSI Standard, ETSI EN 302744 V1.1.4. (2011) Std. ETSI EN 302744 V1.1.4., January 2001.

[21] J. J. van de Beek, M. Sandell, and P. O. Börjesson, "ML estimation of time and frequency offset in OFDM systems," IEEE Tranactions on Signal Processing, vol. 45, pp. 1800$1805,1997$.

[22] B. Yang, K. B. Lataief, R. S. Cheng, and Z. Cao, "Timing recovery for OFDM systems," IEEE Journal on Selected Area in Communications, vol. 18, pp. 2278-2291, November 2000.

[23] M. Bhuiyan, E. Lohan, and M. Renfors, "Code tracking algorithms for mitigating multipath effects in fading channels for satellite-based positioning," EURASIP Journal on Advances in Signal Processing, vol. 2008, no. 1, pp. 1-17, 2008.

[24] G. A. McGraw and M. S. Braasch, "GNSS multipath mitigation using gated and high resolution correlator concepts," in Proceedings of the 1999 National Technical Meeting of The Institute of Navigation, San Diego, U. S. A., January 25 - 27 1999 , pp. $333-342$.

[25] T.-D. Chiueh and P.-Y. Tsai, OFDM Baseband Receiver Design for Wireless Communications. IEEE Wiley and Son (Asia), 2007.

[26] S. H. Mller-Weinfurtner, J. F. Rler, and J. B. Huber, "Analysis of a frame and frequency synchronizer for (bursty) OFDM," in Proc. Globecom98 Commun. Theory Mini-Conf., 1998.

[27] S. M. Kay, Fundamentals of Statistical Signal Processing: Estimation Theory. Prentice Hall, 1993.

[28] H. Minn, V. K. Bhargava, and K. B. Letaief, "A robust timing and frequency synchronization for OFDM systems," IEEE Transactions on Wireless Communications, vol. 2, no. 4, pp. 822-839, July 2003. 